\title{
Engineering Division
}

To be presented at the International Symposium on

Computer Assisted Radiology (CAR '87),

Berlin, Germany, July 1-4, 1987, and to be

published in the Proceedings

THE HIGH SENSITIVITY OF THE MAXIMUM LIKELIHOOD

ESTIMATOR METHOD OF TOMOGRAPHIC

IMAGE RECONSTRUCTION

J. Llacer and E. Veklerov

January 1987

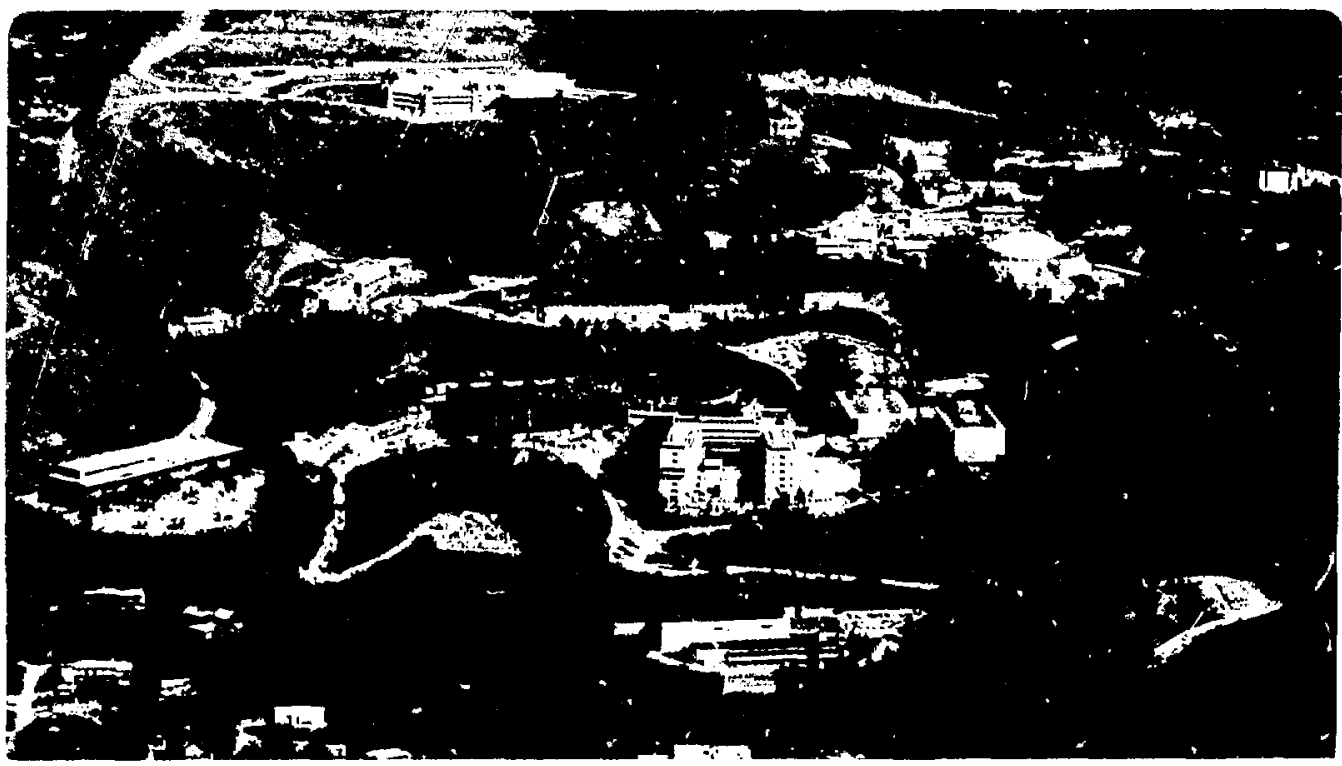

Prepared for the U.S. Department of Energy under Contract DE-AC03-76SF00098 


\begin{abstract}
LEGAL NOTIK:E
This book was prepared as an account of work sponsored by an agency of the United States Government. Neither the United States Covernment nor any agency thereof, nor any of their employees, makes any warranty, express or implied, or assumes any legal liability ar responsibility for the accuracy', completeness, or usefulness of any information, apparatus, product, or process disclosed, or represents that its use would not infringe privately owned rights. Reference herein to any specific commercial product, process, or service by trade mane, trademark, manufacturer, or otherwise, does not necessarily constitute or imply its endorsement, recommendation, or favoring by the United States Government or any agency thereof. The views and opinions of authors expressed hercin do not necessitrily state or reflect those of the United States Covernment or any agency thereof.
\end{abstract}


JORGE LLACER and EUGENE VEKLEROV

Lawrence Berkeley Laboratory

I BL -21874

Mailstop 29-100

University of California

Berkeley, CA 94720 U.S.A.

DE87 007612

Summary

In recent work we have shown that PET images obtained by the MLE iterative method of image reconstruction converge towards strongly deteriorated versions of the original source image. In the present work we show that the image deterioration is caused by an excessive attempt by the algorithm to match the projection data with high counts and that we can modulate this effect. We compare a source image with reconstructions by filtered backprojection and by the MLE algorithm and show that the MLE images can have similar noise to the filtered backprojection images at regions of high activity and very low noise, comparable to the source image, in regions of low activity, if the iterative orocedure is stopped at an appropriate point.

\section{Introduction}

After the Maximum Likelihood Estimator (MLE) method of image reconstruction was proposed by Shepp and Vardi ${ }^{1}$ for emission tomography, it has often been observed that continuation of the iterative process beyond a certain point results in strong image deterioration. Starting from the original activity distribution shown in $\mathrm{Fig}$. 1 , we recently calculated images in a $128 \times 128$ pixel image plane by a random process ${ }^{2}$. These images are called "source images". Using a matrix of detection probabilities calculated for one 512-detector ring of the ECAT-III instrument at UCLA ${ }^{3}$, projection data were obtained again by a random process. The data were then used as input to the MLE algorithm. By using the same matrix of detection probabilities in the reconstruction as in the source image generation we avoided questions regarding accuracy of a particular matrix to define an instrument accurately. We looked exclusively at the behavior of the MLE algorithm.

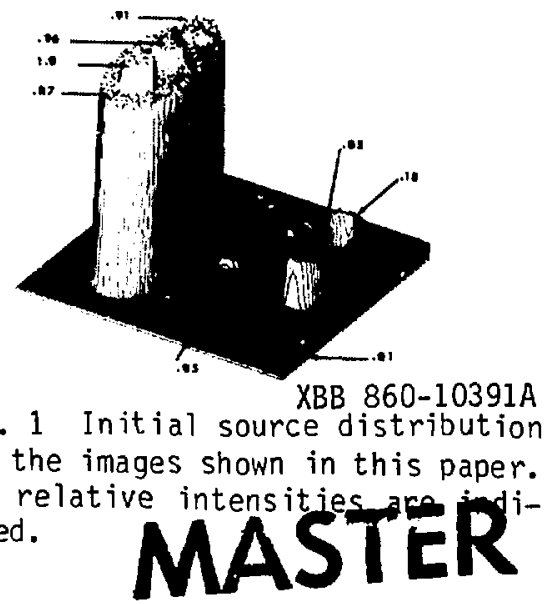


In the work of Ref. 2 we were able to confirm the process of image deterioration and study the convergence characteristics of the MLE algorithm. Figure 2 shows the $\log$ likelihood for the images obtained at different numbers of iterations for a source image with 2 million counts (2M). The horizontal line indicates the likelihood for the true source image. The conclusions from the previous work can be summarized as: 1) the MLE algorithm actually converges towards an image that maximizes the likelihood that the initial projection data would have come from a source distribution corresponding to the obtained image, 2) the asymptotic maximum likelihood image can be a very deteriorated version of the original source distribution, although the quality of the reconstruction increases as the number of counts in the projection data increases, and 3 ) the original source image is not a maximum likelihood image for the projection data. Further data, images and discussion are given in Ref. 2 .

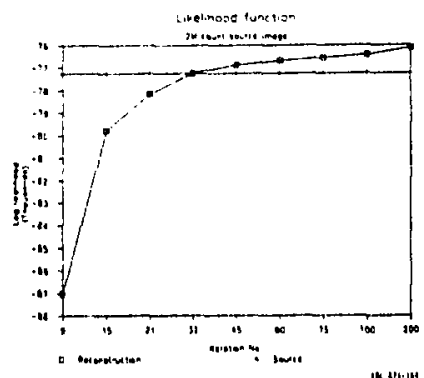

Fig. 2 Log likelihood that the image obtained at a certain number of iterations has yielded that certain set of projection data used as ingut for the reconstruction. The horizontal line indicates the likelihood for the $2 M$ count source that truly generated the input data.

From the above findings one could conclude that the ME algorithm does not have much future in medical tomography, since reconstructions converge towards images that could be sufficiently different from the source image to lead to false diagnosis. It is clear, however, that images obtained with a moderate number of iterations appear to be good representations of the source inage. He inave now quantified the rioise in different parts of the reconstructed images and compared them with the Filtered Backprojection (FBP) method. We have understood the causes of image deterioration and controlled it within a certain range. The analysis leads us to the conclusion that the MLE algorithm can yield excellent images with very low noise in regions of low counts if used properly.

\section{Reconstruction Procedure}

Based on our understanding of the properties of the MLE algorithm, we felt it would be important to incorporate a method of changing the weight given 
to tubes $d$ in the process of maximization. The notation of Shepp and Var$\mathrm{di}^{1}$ is used throughout this paper. We call this modified method the Weighted Likelihood Estimator (WLE). We seek to maximize the function:

$$
W(\lambda)=W P\left(n^{*}|\lambda\rangle=\prod_{d=1}^{D}\left[e^{-\lambda^{*}(d)} \lambda^{*}(d)^{n^{*}(d)} / n^{*}(d) !\right]^{s \cdot n^{*}(d)+t}\right.
$$

where $n *(d)$ is the number of counts detected in a tube $d$, and $\lambda^{*}(d)$ is the projection into tube $d$ of the reconstructed image. With $s=0$ and $t=1$, the function $W$ is identical to the likelihood function $L$ of Ref. 1 . Keeping $t=1, s>0$ will give higher weight to those tubes that have higher number of counts, while making $s<0$ will decrease their weight. Unlike $L$ of Ref. 1 , $W L(\lambda)$ does not have the meaning of the probability to obtain the projection data $n^{*}$ from the image $\lambda^{\star}$. However, if $s$ is small, both $L$ and $W L$ increase monotonically hy iteration. The iterative formula for the maximization of Eq. 1, obtained by a method similar to that of Ref. 1 is the following:

$$
\lambda^{\text {new }}(b)=\lambda^{\text {old }}(b)\left[1+\sum_{d=1}^{D}\left[s \cdot n^{*}(d)+t\right] p(b, d) \frac{n^{*}(d)-1}{\sum_{b^{\prime}=1}^{B} \lambda^{0 l d}\left(b^{\prime}\right) p\left(b^{\prime}, d\right)}\right]
$$

\section{Results of Reconstructions}

We have used a source image with $2 M$ counts based on the activity distribution shown in Fig. 1. Figure $3 a$ shows a cut through the source image. Figure 3b shows the FBP results with the Shepp-Logan filter and Figs. 3c, $d$ and $e$ show the results from the unmodified MLE at 9, 32 and 200 iterations, respectively. We have also carried out reconstructions with the WLE for values of $s=0.0025$ and -0.0015 . It is observed that the onset of image deterioration in regions of high activity comes early in the first case and is delayed in the second case. No substantial differences are observed in regions of low activity.

\section{Evaluation of Results}

We have defined two regions, 1 and 2 , in the source image representing high (1.0) and low (0.05) activity regions, respectively, as shown in Fig. 4. The mean values of the reconstructions and the standard error from the mean have been calculated in each zone. Figures $5 a$ and $b$ show error plots for the two regions. In region 1 we observe that the error 
for the FPB is a factor of 2 higher than the source image error, normal for that method of reconstruction. For the WLE method, we see a substantial influence of the parameter $s$ on the iteration number at which the std. error is equal to that of the FBP method. In region 2, the error of the FBP is $\sim 0.05$, of the same magnitude as the signal, while the WLE results remain under 0.01 (near the source noise) up to iterations 40 to 60 , depending weakly on parameter s. Even at iteration 200, with $s=0$ or -0.0015 the std. error remains under 0.02 , with a marked superiority over the FBP method.

\section{Discussions and Conclusions}

The behavior of the WLE reconstructions when the parameter $s$ is changed indicates that the progressive deterioration of the images is due to an attempt by the algorithm to match excessively well projection data with high number of counts. We have made the observation earlier 2 that the MLE algorithm gains more likelihood by matching projection data with low number of counts than with high counts. We are now finding that, due to the imperfect nature of the count limited projection data, the MLE method still tries too hard to match regions of high activity and yields unacceptable images if allowed to it- She erate without limits. Considering that it is possible to obtain images tions. e) ditto, 200 iterations.

Fig. 3 Cuts through source and reconstructed images. a) source image with 2 million counts. b) reconstruction by filtered backprojection, Shepp-Logan filter. c) reconstruction by maximum likelihood estimate, 
with the MLE that have similar noise as the $F B P$ in regions of high activity, and much lower noise in regions of low activity when the iterations are stopped at an appropriate point, it appears that it would be fruitful to define a criterion for iteration stopping based on statistical considerations. We are continuing work in Fig. 4 Source distribution showing that direction.

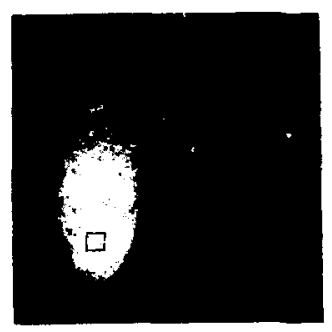

XBB 871-482 Regions 1 and 2 for noise evaluation.
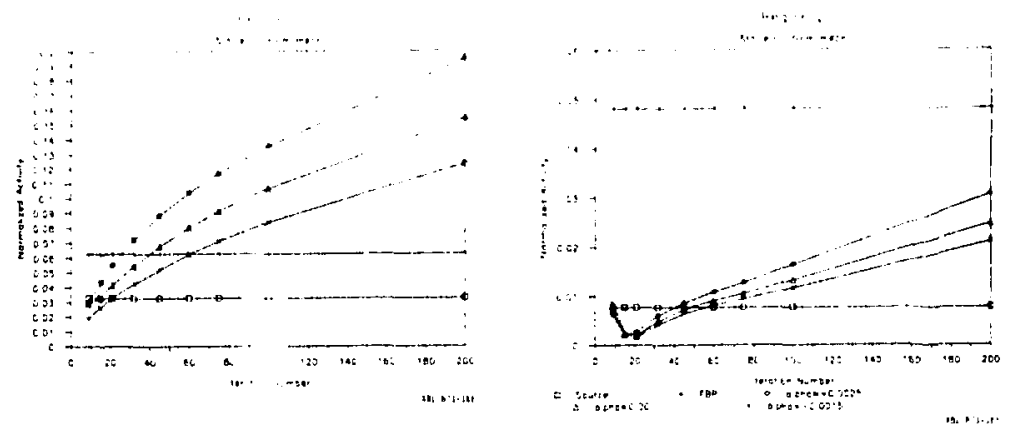

Fig. 5 Plots of standard error from the mean as a function of iteration number for different values of parameter $s$ in Eqs. 1 and 2. a) for re-gion 1 , with high counts $(1.0)$. b) for region 2, with low counts $(0.05)$.

\section{Acknowledgment}

This work has been supported by a grant from the National Cancer Institute (CA-39501) and the U.S. Department of Energy under Contract No. DE-AC03-76SF00098.

\section{References}

1. Shepp, L.A. and Vardi, Y.: Maximum Likelihood Reconstruction for Emission Tomography, IEEE Trans. Med. Imaging, MI-1, No. 2, 113-121 (1982).

2. Llacer, J., Veklerov, E. and Hoffman, E.J.: On the Convergence of the Maximum Likelihood Estimator Method of Tomographic Image Reconstruction, Proc. of Conf. on Medical Imaging, Newport Beach, CA (1987). SPIE Vol. 767 (1987).

3. Hoffman, E.J., Ricci, A.R., Van der Stee, L.M.A.M. and Phelps, M.E.: ECAT-III, Basic Design Considerations, IEEE Trans. Nucl. Sci., NS-30, No. 1, 729-733 (1983). 


\section{DISCLAIMER}

This report was prepared as an account of work sponsored by an agency of the United States Government. Neither the United States Government nor any agency thereof, nor any of their employees, makes any warranty, express or implied, or assumes any legal liability or responsibility for the accuracy, completeness, of usefulness of any information, apparatus, product, or process disclosed, or represents that its use would not infringe privately owned rights. Reference herein to any specilic commercial produrt, process, or service by trade name, trademark, manufacturer, or otherwise does not necessarily constitute of imply its endorsement, recummendation, or favoring by the United States Government or any agency thereof. The views and opinions of authors expressed herein do not necessarily state or reflect these of the United States Government or any agency thereof. 
This report was done with support from the Department of Energy. Any conclusions or opinions expressed in this report represent solely those of the author(s) and not necessarily those of The Regents of the University of California, the Lawrence Berkeley Laboratory or the Department of Energy.

Reference 10 a company or product name does not imply approval or recommendution of the product by the University of Californis or the U.S. Department of Energy to the exclusion of others that may be suitable. 